\title{
¿Nuevas o viejas masculinidades? El rol masculino dominante entre los adolescentes españoles ${ }^{1}$
}

\author{
New or old masculinities? The dominant male role of Spanish \\ adolescents
}

\author{
Mayra Martínez Avidad \\ Universidad Camilo José Cela, España \\ mmartinez@ucjc.edu
}

\section{Alba Pérez López}

Universidad Camilo José Cela, España

albaper.lopez@gmail.com

Recibido / Received: 04/03/2020 Aceptado / Accepted: 23/03/2020

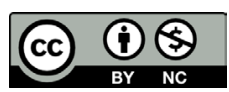

\section{RESUMEN}

A pesar de los nuevos valores en torno a la igualdad de género, no queda claro que éstos hayan conseguido cambios sustantivos en el modelo de masculinidad de las nuevas generaciones. La presente investigación se propone indagar en qué medida ha cambiado el rol de género de unos varones que han crecido y han sido educados en una sociedad supuestamente más igualitaria. Para ello se ha utilizado una metodología cualitativa basada en grupos de discusión y entrevistas semi-estructuradas con adolescentes que se encuentran en los últimos cursos de Educación Secundaria Obligatoria. En general se observa una persistencia del rol masculino patriarcal, con algunas variaciones también destacables, consecuencia de algunos cambios sociales que han transformado las relaciones entre sexos.

Palabras clave: Masculinidad hegemónica; nuevas masculinidades; género; roles sociales; adolescentes.

\section{ABSTRACT}

Despite the new values of gender equality, it is not clear that they had been able to generate substantive changes in the model of masculinity of new generations. This research aims to determine to what extent the gender role of boys that have been grown and have been educated in a supposedly more egalitarian society, have changed. For this purpose, we use a qualitative methodology base on focus groups and semistructured interviews with adolescents that are in the last courses of secondary education. In general, we observe a persistence of the patriarchal male role, with also some remarkable variations, consequence of some social changes that have transformed relations between men and women.

Keywords: Hegemonic masculinity; new masculinities; gender; social roles; adolescents.

*Autor para correspondencia / Corresponding author: Mayra Martínez Avidad mdiaz-bu@ucm.es

Sugerencia de cita / Suggested citation: Martínez Avidad, M. y Pérez López, A. (2020). ¿Nuevas o viejas masculinidades? El rol masculino dominante entre los adolescentes españoles. Revista Española de Sociología, 29 (3, supl. 1), 171-189. https://doi.org/10.22325/fes/res.2020.63

1 Esta investigación se basa parcialmente en el trabajo fin de master sobre masculinidades realizado por Alba Pérez López bajo la dirección de Mayra Martínez Avidad. 


\section{INTRODUCCIÓN}

El debate en torno a la construcción social del rol femenino y las desigualdades resultantes ha copado la esfera pública en los últimos años. Ello ha contribuido a la deconstrucción de muchas definiciones sexistas de lo femenino que han desencadenado, a su vez, importantes transformaciones sociales y nuevas problemáticas en las relaciones entre los sexos (Beck y Beck-Gernsheim, 2001; Bauman, 2005; Castells y Subirats, 2007). Sin embargo, quizá debido a que tradicionalmente los estudios de género han abordado el tema del rol como modo de hacer evidentes las estructuras que contribuían a reproducir el poder hegemónico heteropatriarcal y sus jerarquías de dominación, socialmente se ha descuidado la atención a las paradojas y conflictos inherentes a la construcción social de lo masculino. Paralelamente a los mecanismos de construcción social del rol de género femenino, las instituciones socializadoras (familias, escuelas, medios de comunicación, Internet e industrias culturales) también construyen, a través de discursos y prácticas sociales, los preceptos normativos que encarnan la manera más honorable y legítima de ser hombre, deslegitimando otras posibles (Bonino, 2003). Estas coordenadas simbólicas sobre qué es y qué no es un hombre -elementos constitutivos del proceso de masculinizaciónproducen "ese artefacto social llamado un hombre viril" (Bourdieu, 2000: 21).

Con todo, los estudios sobre masculinidades son cada vez más numerosos y comienzan a ser conocidos los costes y consecuencias negativas asociadas al rol masculino dominante. Gran parte de la investigación se ha ocupado, de hecho, de los patrones de agresión, criminalidad, prácticas en el cuidado de la salud, perjuicios emocionales y psicológicos, así como las frecuentes tensiones que rodean al rol de género masculino (Connell, 2000; Bonino, 2002; Mac an Ghaill y Haywood, 2012); investigaciones que, por otro lado, también están contribuyendo al surgimiento de iniciativas institucionales orientadas al desarrollo de 'nuevas masculinidades' (Azpiazu Carballo, 2017). Ciertamente, la masculinidad hegemónica no es la normal estadísticamente (solo una minoría la promulga) pero sigue siendo normativa en el sentido que encarna esa forma más genuina de ser hombre y requiere que todos los hombres se posicionen en relación a ella (Connell y Messerschmidt, 2005). Este ideal normativo es además uno de los factores básicos en la reproducción/ perpetuación de las desigualdades de género (Bonino, 2003).

Dado que las definiciones sociales de lo masculino se construyen fundamentalmente en oposición a lo femenino, cabe esperar que algunas de las transformaciones más recientes en torno a las representaciones y prácticas sociales de las mujeres, hayan provocado también variaciones en la denominada 'masculinidad hegemónica'. Parece que muchos hombres jóvenes, que hoy esperan que las mujeres rechacen relaciones patriarcales, están adoptando un cierto 'igualitarismo pragmático' (Connell y Messerschmidt, 2005). Algunas investigaciones sí sugieren ciertas variaciones sutiles y fragmentaciones en la masculinidad dominante de las nuevas generaciones, sin que ello implique que los nuevos modelos que algunos autores denominan 'híbridos' (Bridges y Pascoe, 2014)- demuestren cambios realmente sustantivos, ni resulten más igualitarios (Azpiazu Carballo, 2017).

El ideal masculino parece haber variado poco en términos generales, a pesar de haber quedado maltrecho por el nuevo ideal de igualdad y los rápidos cambios en las relaciones de género. De ahí que los varones se sientan a menudo amenazados y confusos y respondan a estas demandas contradictorias con una pluralidad de actitudes y posicionamientos a veces ambivalentes (García García, 2010). Un ejemplo de estas paradojas lo encontramos en el hecho de que, al mismo tiempo que cada vez más jóvenes se declaran feministas (Ballesteros, et al., 2019), la violencia de género crece especialmente entre los chicos más jóvenes. De hecho, las denuncias por violencia machista que más se elevaron en 2018 frente al año anterior, correspondieron al tramo 
de 18 a 19 años (un 14,1\% más) (Valdés, 2019). Los valores de igualdad respaldados por las nuevas generaciones también casan mal con las estadísticas de muertes accidentales entre los varones jóvenes. En el tramo de 19 a 23 años esta tasa supone hasta tres veces la registrada por las chicas de la misma edad, lo que puede atribuirse a los mandatos de competitividad y asunción de riesgos del modelo masculino tradicional (García García, 2010).

Partiendo de este escenario complejo, pretendemos conocer qué rasgos concretos del ideal masculino hegemónico siguen vigentes entre los jóvenes y cuáles han podido sufrir más erosión como consecuencia de los cambios sociales, el auge del feminismo y la llamada 'crisis del patriarcado'. Paralelamente, se pretende ahondar en las imposiciones contradictorias a las que se enfrentan los jóvenes en los diferentes contextos de interacción, las acciones que realizarían de forma consciente para adaptarse al rol dominante, así como el coste percibido de cumplir con el ideal masculino.

Para llevar a cabo la investigación se ha utilizado una metodología cualitativa basada en grupos de discusión y entrevistas semi-estructuradas con adolescentes que se encuentran en los últimos cursos de Educación Secundaria Obligatoria.

\section{MARCO TEÓRICO}

Los procesos relacionados con la adquisición y reproducción de los roles de género han sido ampliamente abordados por diferentes enfoques y campos de estudio. Para la tradición sociológica que va de Durkheim al estructural-funcionalismo de Parsons, la propia existencia de la sociedad depende de la interiorización de los roles sociales -entre ellos los de género- a través de los cuales los individuos interiorizan los valores, normas y jerarquías presentes en la sociedad. El constructivismo social de Berger y Luckmann y las contribuciones de Goffman al interaccionismo simbólico, añadieron al análisis de los roles sociales la relación dialéctica mediante la cual el orden social, no sólo es reproducido desde las propias instituciones sociales, sino que éste es al mismo tiempo generado localmente en las interacciones y prácticas discursivas diarias. Por su parte, la corriente post-estructuralista, la literatura feminista y la sociología crítica, en la que se inscriben autores como Foucault, Butler o Bourdieu, añaden un elemento fundamental al análisis: las relaciones de poder preexistenes. De esta manera el poder, que está presente en todas las relaciones, estructuras sociales y discursos, condicionan esa matriz generativa de orden social, ese sistema normativo que organiza el mundo y reproduce las jerarquías sociales, al cual denominamos 'rol de género'. En la revisión teórica recogemos precisamente estas aportaciones que consideramos más significativas a la hora de comprender los procesos involucrados en la adquisición, interiorización y reproducción del rol de género masculino. A este recorrido por la literatura académica clásica y contemporánea sumamos, como no, algunas aportaciones fundamentales al estudio de las masculinidades desde el punto de vista sociológico.

\section{Adquisición, interiorización y reproducción del rol de género masculino}

Tal y como ha puesto de relieve la ciencia social, el rol de género comienza a ser adquirido desde casi el momento del nacimiento. Es durante la socialización primaria, y fundamentalmente a través del lenguaje, cómo diversos esquemas motivacionales e interpretativos se internalizan. "Por ejemplo, el tener que actuar como un muchachito valiente y el suponer que los muchachitos se dividen en valientes y cobardes" (Berger y Luckmann, 1968: 169). Según Berger y Luckmann, estos esquemas proporcionan al niño prescripciones institucionalizadas para aplicarse en la vida cotidiana y establecen las 
diferencias entre la identidad propia y la de las niñas, al mismo tiempo que enseñan el porqué de estas prescripciones. Resulta especialmente llamativo que el aparato legitimador de los rasgos masculinos hegemónicos haya sido habitualmente una apelación al esencialismo: 'Hay que ser valiente porque así son los hombres de verdad'. Precisamente debido a esta tendencia a conferir un 'status ontológico' a lo que es una tipificación construida por los seres humanos, resulta frecuente que los niños establezcan una fuerte identificación con los roles de género socialmente atribuidos.

También para Butler (2001) el uso del lenguaje es un mecanismo de control sobre las creencias, actitudes y conductas de los sujetos en relación a su identidad sexual. La autora recurre, así, al acto perlocucionario definido por Austin (1971), según el cual, algunos enunciados (los llamados performativos) producen por sí mismos efectos en los sentimientos, predisposiciones y acciones de los individuos, actuando, por tanto, sobre la realidad. A este tipo de actos del habla se une la fuerza ilocucionaria de un repertorio de actos corporales y otros medios discursivos que necesitan de la repetición ritualizada para desplegar sus efectos. En estos supuestos se fundamenta la conocida 'teoría de la performatividad' de Butler², también deudora de Derrida (1988), para quien la posibilidad de diseminar los códigos discursivos, y que éstos sean repetidos indefinidamente por terceros en diferentes contextos (propiedad de iterabilidad), les convierte en marcos reguladores en sí mismos más allá de la intención de los sujetos que enuncian.

Son estos discursos regulativos ampliamente compartidos y diseminados, los que articulan las construcciones genéricas de los roles de género, los cuales acaban además naturalizándose y produciendo la ilusión de esencia (Duque, 2010). A continuación, vemos algunos ejemplos de estas prácticas discursivas que establecen conductas acordes con las prescripciones culturales de la identidad masculina:

Podemos observar, a manera de ilustración, las prácticas de crianza occidentales en las cuales desde que nace el niño, tiene un lugar y un papel predeterminado en el mundo: su ropa será azul, sus juegos estarán relacionados con la fuerza, la competencia y el poder (armas, carros, fútbol, caballos de madera, etc.); tendrá menos restricciones en su movimiento (no usará vestidos largos e incómodos, faldas ni sandalias que por ejemplo le impidan subir a un árbol), el trato de los hombres de la casa hacia él tendrá cierto nivel de fuerza y temple; y por su puesto se le prohibirá en lo posible llorar ("los hombres no lloran") o ser "afeminado" (maquillarse, jugar con muñecas o con utensilios de cocina), así como expresar atracción o sentimiento estético por otros niños. (Duque, 2010: 88)

Este tipo de prácticas discursivas continuadas ejercerán tal efecto en el individuo, que en no pocas ocasiones el imperativo de amoldamiento social consigue vencer al substrato biológico:

Por ejemplo, un hombre debe vencer su temor instintivo a la muerte mediante el valor (...). Similarmente, un hombre puede obligarse a ejercer sus funciones sexuales venciendo la resistencia inerte de su saciedad fisiológica, con el propósito de mantener su identidad como modelo de hombría". (Berger y Luckmann, 1968: 225)

Ciertamente, el debate biología vs ambiente se torna superfluo cuando nos percatamos del poder de las formaciones socio-culturales a la hora de modular algunas funciones biológicas ${ }^{3}$. Por otro lado, las posiciones biologistas que relacionan el género con el sexo del individuo pierden también preeminencia cuando la evidencia etnológica da cuenta de

2 La posición de Butler va un paso más allá del construccionismo social al defender que, no sólo el género, también el propio binarismo sexual es producto de las configuraciones socio-históricas. De hecho, para la filósofa no es correcto definir el género como una interpretación cultural del sexo, pues es el propio rol de género -entendido como constructo social que se perpetúa y reproduce mediante una serie de discursos- el que prescribe una (hetero)normatividad sexual.

3 Berger y Luckmann (1968) recuerdan que la socialización impone límites a muchas funciones del organismo como la sexualidad o la nutrición, hasta el punto de que el individuo exitosamente socializado es incapaz de realizar conductas consideradas 'impropias'. 
la variabilidad de los roles asumidos por mujeres y hombres en los diferentes contextos sociales y culturales. Un ejemplo serían las prácticas sexuales socialmente normalizadas en la Antigua Grecia que eluden una de las tipificaciones básicas de la masculinidad hegemónica más reciente: el de la heterosexualidad. En su obra ya clásica, Historia de la Sexualidad (2003), Foucault muestra cómo en la sociedad griega de la época, ni siquiera existía la concepción actual de homosexualidad. Regidos por un código ético diferente, para los griegos lo fundamental no era la preferencia de mujeres frente a muchachos, sino el de dominar y no ser dominado por los propios deseos (moderación) y el de evitar la pasividad sexual.

Sin embargo, es precisamente este "trabajo colectivo de socialización de lo biológico y de biologización de lo social", lo que según Bourdieu (2000: 6), contribuye a "invertir la relación entre las causas y los efectos y a hacer aparecer una construcción social naturalizada como el fundamento natural de la división arbitraria" entre hombres y mujeres; lo cual legitima, al mismo tiempo, las desigualdades inherentes a esa división. Bourdieu (2000) sostiene que la dominación masculina consiste, de hecho, en este proceso de familiarización con los discursos, prácticas y espacios que reproducen los esquemas fundamentales de la visión androcéntrica del mundo. La hegemonía masculina se inscribe, así, en las estructuras sociales otorgando a los hombres esa posición privilegiada en términos de capital simbólico y valor social que también las mujeres incorporan a sus estructuras cognitivas a través del 'habitus'. El mecanismo de reproducción de esta jerarquía está, de este modo, fácilmente asegurado: debido a su posición privilegiada en la estructura social, los hombres no sólo disponen de más poder a la hora de seguir conformando las maneras de organizar la sociedad y las definiciones de la realidad favorables, sino que cuentan, además, con la complicidad de las víctimas de la dominación simbólica.

\section{Rasgos asociados a la masculinidad hegemónica tradicional y sus efectos negativos}

Las definiciones oficiales del rol de género que fluyen desde las instituciones centrales de la sociedad suelen englobar los atributos, normas y valores que consiguen mayor consenso y aprobación social al expresar valores y jerarquías socialmente aceptadas en un momento histórico dado. Este rol hegemónico constituye la generalidad de la norma o el 'rol general' que alude, al mismo tiempo, a una identidad colectiva, y que el niño incorpora subjetivamente en el proceso de socialización primaria. Es a través de este proceso de abstracción de los roles de otros significantes -lo que Mead (1973) denomina 'el otro generalizado'- cómo la sociedad ejerce control sobre sus miembros individuales. Adicionalmente, para corregir cualquier posible desviación de la norma oficial, la sociedad también cuenta con una serie de mecanismos de control: desde la presión ejercida por los otros niños socializados exitosamente, hasta los dispositivos terapéuticos para la 'reparación' de la identidad desviada (Berger y Luckmann, 1968).

Los mandatos de la masculinidad clásica, sobre la que se ha teorizado ampliamente en las últimas dos décadas, pueden resumirse en una serie de aspectos socialmente identificables:

Un hombre es quien no muestra sus sentimientos, quien ejerce autoridad por todos los medios, quien hace uso autolegitimado de la violencia, quien no tiene contacto sexual o afectivo con otros hombres, quien se pone en riesgo para mostrar su valentía. (Azpiazu Carballo, 2017: 34)

Basándonos principalmente en el trabajo realizado por Bonino (2003), al que incorporamos el rasgo de 'Heterosexualidad activa' recogido por Muñoz Sánchez (2017), en la Tabla I resumimos los rasgos o dimensiones propias de la masculinidad hegemónica tradicional, así como los preceptos normativos y las conductas y actitudes asociadas a cada uno de ellos. 
Tabla I. Rasgos o dimensiones de la masculinidad hegemónica tradicional, mandamientos y conductas asociadas

\begin{tabular}{|c|c|c|}
\hline $\begin{array}{l}\text { Dimensiones de } \\
\text { la masculinidad } \\
\text { hegemónica tradicional }\end{array}$ & Mandamientos básicos & Conductas/actitudes \\
\hline $\begin{array}{l}\text { Autosuficiencia e } \\
\text { individualidad }\end{array}$ & $\begin{array}{l}\text { Valerse por uno mismo; ser inde- } \\
\text { pendiente y no apoyarse en nadie; } \\
\text { destacar como diferente y superior. }\end{array}$ & $\begin{array}{l}\text { Autoconfianza; autonomía; indivi- } \\
\text { dualismo; tendencia a demostrar } \\
\text { superioridad. }\end{array}$ \\
\hline $\begin{array}{l}\text { Trascendencia, éxito } \\
\text { y prestigio social }\end{array}$ & $\begin{array}{l}\text { Lograr éxito y poder; alcanzar una } \\
\text { posición social relevante a través } \\
\text { del ámbito profesional; obtener } \\
\text { reconocimiento social. }\end{array}$ & $\begin{array}{l}\text { Búsqueda del éxito profesional; } \\
\text { ambición y competitividad laboral. }\end{array}$ \\
\hline $\begin{array}{l}\text { Trabajador/proveedor } \\
\text { y responsable de } \\
\text { su familia }\end{array}$ & $\begin{array}{l}\text { Ser responsable de su mujer e } \\
\text { hijos; ser capaz de ofrecerles } \\
\text { protección y sustento. }\end{array}$ & $\begin{array}{l}\text { Asunción del rol dominante de } \\
\text { cabeza de familia; inclinación } \\
\text { a satisfacer las demandas que } \\
\text { impone su carrera profesional y } \\
\text { menor preocupación por aspec- } \\
\text { tos domésticos y aquellos } \\
\text { relacionados con la crianza de los } \\
\text { hijos }\end{array}$ \\
\hline Heroísmo y violencia & $\begin{array}{l}\text { Ser fuerte, valiente y luchador; } \\
\text { dominar y no ser dominado; no } \\
\text { dejarse avasallar; resistir el dolor } \\
\text { y las adversidades sin quejarse ni } \\
\text { mostrar debilidad. }\end{array}$ & $\begin{array}{l}\text { Búsqueda de hazañas y proezas; } \\
\text { impulsividad, afrontamiento del } \\
\text { riesgo y espíritu de aventura; } \\
\text { aguante para soportar dolor } \\
\text { y superar sufrimiento; dureza } \\
\text { emocional y aplomo con inhibición } \\
\text { del miedo, la debilidad o el llanto; } \\
\text { emocionalidad distante y uso de } \\
\text { la violencia como instrumento } \\
\text { legítimo. }\end{array}$ \\
\hline $\begin{array}{l}\text { Superioridad sobre } \\
\text { las mujeres }\end{array}$ & $\begin{array}{l}\text { No dejarse someter por las mujeres; } \\
\text { destacar sobre ellas en todos los } \\
\text { ámbitos con excepción a las áreas } \\
\text { típicamente femeninas. }\end{array}$ & $\begin{array}{l}\text { Demostrar y pugnar por el dominio } \\
\text { en la relación de pareja; relegar a } \\
\text { la mujer como pasiva admiradora; } \\
\text { entenderse con mayor derecho al } \\
\text { protagonismo que las mujeres y } \\
\text { ostentarlo. }\end{array}$ \\
\hline $\begin{array}{l}\text { Heterosexualidad } \\
\text { activa }\end{array}$ & $\begin{array}{l}\text { Conquistar a un gran número de } \\
\text { mujeres y relacionarse con ellas } \\
\text { sexualmente; no albergar ningún } \\
\text { atributo, rasgo, ni actitud que pueda } \\
\text { considerarse femenina; no relacio- } \\
\text { narse afectiva o sexualmente con } \\
\text { otros hombres; demostrar potencia } \\
\text { sexual superior. }\end{array}$ & $\begin{array}{l}\text { Rol sexual activo y exhibición } \\
\text { de gran potencia sexual como } \\
\text { demostración de virilidad. }\end{array}$ \\
\hline
\end{tabular}

Fuente: Adaptado de Bonino (2003)

Las creencias que componen esta matriz de género masculino llevan sin embargo asociadas importantes consecuencias negativas para la vida de los varones. Es la llamada 'problemática silenciada' de la masculinidad hegemónica. Una de las coordenadas matrices, el de 'autosuficiencia e individualidad', conlleva por ejemplo comportamientos como la represión emocional, la negación de ciertos vínculos afectivos, el rechazo a la ayuda de otros y el afrontamiento en solitario de los problemas (Navarro, 2004). En los peores casos 
estas autoexigencias pueden generar trastornos psicológicos que, unido a la imposibilidad de pedir ayuda, pueden derivar en suicidio, cuya frecuencia en nuestro país, casi triplica a la de las mujeres (2.718, frente a 961 en 2017) (INE, 2019).

Por su parte, el precepto de 'transcendencia y éxito' genera estrés, miedo al fracaso, conductas de auto-explotación (adicción al trabajo) y 'síndrome de burnout' (González, 2006). Al mismo tiempo, los varones experimentan frecuentes sentimientos de inadecuación y fracaso si no pueden cumplir debidamente con su 'papel de proveedor' como padres y cabeza de familia (Rodríguez, 2014). Los efectos de la división sexual del trabajo y la desigual distribución de los cuidados -causante de que ellas padezcan una menor proyección social, laboral y económica- genera su contrapartida en los hombres: en caso de divorcio, mayor tasa en pérdidas de custodias y obligación de abandonar el hogar familiar. Aunque la custodia compartida se ha triplicado en relación a hace una década, aún sigue siendo mayoritaria la concedida en exclusiva a la madre (el 61,6\% en 2018), frente a las infrecuentes custodias obtenidas por el padre (4,2\% en 2018) (INE, 2019).

Pero quizá el mandato masculino que más estragos causa entre los hombres es la demostración de 'heroísmo y el uso de la violencia'. El ideal heroico está asociado a competitividad y conductas de riesgo que derivan en accidentes deportivos y de tráfico, transgresión de normas, peleas y conductas delictivas y antisociales. Resulta indicativo que los hombres fallecidos en accidentes de tráfico en España en 2017 fueran más del triple que las mujeres (INE, 2019). Ese año, casi el $80 \%$ de los condenados por algún tipo de delito fueron también varones (INE, 2018). Todo ello, unido a otras cuestiones, como una mayor frecuencia de adicciones (Ministerio de Sanidad, Servicios Sociales e Igualdad, 2019), puede estar también relacionado con una menor esperanza de vida. En 2018, la brecha fue de 5,3 años menos de vida para los hombres (INE, 2019). En las cohortes jóvenes la tasa de probabilidad de muerte masculina triplica y llega a cuadriplicar la femenina (García García, 2010). En el ámbito escolar, este tipo de conductas valoradas por los varones coinciden también con un mayor número de sanciones disciplinarias, así como con un mayor abandono y fracaso escolar entre los chicos (Sanz, 2016).

El precepto de 'heterosexualidad activa' del modelo hegemónico ejerce también fuerte presión sobre los hombres. Es ilustrativo que el primer y tercer motivo de burlas y exclusión entre el alumnado, según la percepción del profesorado, sean respectivamente: "ser un chico que parece o se comporta como una chica", y "ser o parecer gay" (Pichardo Galán, et. al, 2015). Paralelamente, los jóvenes sufren la presión de ser activos sexualmente y la coacción de los pares por ejercer conductas que reproduzcan la jerarquía masculina (Segato, 2018) ${ }^{4}$. Esto, unido a la cosificación de la mujer en la sociedad, -aspecto en el que también puede estar influyendo la industria del porno, cuyo consumo es mucho más frecuente entre los hombres (Ballester Brage y Orte Socias, 2019)- puede estar en parte relacionado con los numerosos casos de abusos sexuales, producidos casi invariablemente por varones. En 2018 el 98,28\% de los detenidos e imputados por delitos sexuales fueron hombres (INE, 2019).

Finalmente, la necesidad de demostrar 'superioridad sobre las mujeres', en combinación con otros de los mandatos ya mencionados -como el uso legitimado de violencia, o el hecho de que las formas de expresión emocional permitidas se reduzcan prácticamente a manifestaciones de rabia e ira-, parecen determinantes a la hora de explicar las alarmantes cifras de violencia de género en España. Solo en 2018, 48 mujeres fueron asesinadas por sus parejas o exparejas, y se interpusieron 166.961 denuncias por violencia de género (Ministerio de Presidencia, Relaciones con las Cortes e Igualdad, 2019).

4 Segato señala que existe una correspondencia entre este mandato de la masculinidad y la concepción del cuerpo femenino como un terreno explotable y susceptible de ser vejado. 


\section{Contradicciones, paradojas y ambivalencias en la representación del rol de género masculino}

Los esquemas básicos de acción y pensamiento acordes con el rol de género, no permanecen, sin embargo, invariables y estáticos independientemente de los contextos. A través de sus minuciosas descripciones acerca de las interacciones en la vida cotidiana, Goffman (1981) muestra que las actitudes y comportamientos esperados varían según el contexto en el que se desplieguen. En el caso del rol de género masculino resulta evidente, por ejemplo, que no son iguales las características y comportamientos valorados y aceptables en contextos predominantemente masculinos, que en aquellos mixtos en presencia de mujeres. Esto es fácilmente observable en "las 'despedidas de solteros', durante las cuáles pueden exaltarse características comunes que podrían resultar groseras para ellas" (Ramírez, 2011: 253). Del mismo modo, son diferentes las constricciones que impone un contexto homosexual y otro heteronormativo. Un hombre homosexual puede incluso presentar una fachada personal masculina diferente ante sus amigos, que ante su familia y compañeros de trabajo, a quienes aún no ha revelado que es gay (Ramírez, 2011).

Muchas de las rupturas y ambivalencias en las representaciones de los géneros son producto de la propia estructura social. En su ensayo sobre la ambivalencia sociológica (1980) Robert Merton (citado por Martín Criado, 2014), ya señalaba que los roles sociales generan continuamente oscilaciones y dilemas entre los sujetos, pues aquellos pueden, por ejemplo: plantear exigencias contradictorias, entrar en conflicto con otros roles que forman parte de la identidad social del sujeto, o que éste se encuentre con dificultades para cumplirlo en la práctica real. Todo esto es especialmente habitual en lo referente al rol de género. Moen y Orrange (2002) ponen como ejemplo las divergencias entre la demanda propia del rol masculino dominante de ser el sostén familiar, y las demandas simultáneas de sus parejas de participar activamente en el trabajo doméstico y de crianza. Además, las demandas de corresponsabilidad en el trabajo doméstico tropiezan con horarios de trabajo que siguen estructurados alrededor del modelo de 'hombre-proveedor' el cual exige jornadas a tiempo completo (si no más), con permisos de paternidad reducidos ${ }^{5}$ (Moen y Orrange, 2002).

Las contradicciones son también frecuentes en aquellos casos en los que los hombres desempeñan profesiones tradicionalmente femeninas como la de maestro. Así, por ejemplo, aunque por un lado la sociedad reivindica que existan cada vez más maestros de primaria varones, por otro lado, les somete a un escrutinio continuo por si son gais o pedófilos, al mismo tiempo que les considera menos competentes para el cuidado de los niños que a sus compañeras de profesión (Sargent, 2000; Fordice y Nielsen, 2013).

También es habitual la contradicción existente entre los preceptos normativos de 'individualismo' e 'insensibilidad', propios de la masculinidad dominante, y las demandas de las significantes femeninas acerca del cuidado de los vínculos a través de la expresión de los afectos. Algunas investigaciones han mostrado cómo las tensiones son a menudo visibles en las relaciones de pareja heterosexuales. Es común, por ejemplo, que las chicas reprochen a sus parejas la falta de gestos que, según sus propias narrativas amorosas, expresarían atención y cariño, como regalos, sorpresas o expresión del amor en cartas y notas; reproches que, por otro lado, producen sorpresa, malestar y enojo entre los varones (Vicente Olmo, 2018). Otros investigadores han dado cuenta de las ambivalencias surgidas cuando entra en juego el doble estándar de género vigente en el terreno de las prácticas sexuales entre adolescentes. Mientras que el valor social de las chicas se sigue rigiendo por el recato sexual, el de los chicos gira entorno a la promiscuidad, lo cual deriva en que

\footnotetext{
5 Con referencia a los permisos de paternidad hay que señalar, sin embargo, que éstos han evolucionado considerablemente en los últimos años hacia una mayor equidad de género (Jurado y Bueno, 2019) y que, como era de esperar, han probado ser una medida exitosa para fomentar la corresponsabilidad de ambos progenitores en las tareas de cuidado (Meil Landwelind, 2017).
} 
a menudo los varones, "por un lado, premien la poca experiencia sexual de las chicas, y al mismo tiempo, se quejen de ello" (Venegas, 2018: 269).

Muchas de estas paradojas surgen de manera especial cuando los sujetos entran en contacto con otras definiciones posibles del rol procedentes de mundos culturalmente discrepantes (Berger y Luckmann, 1968). Las discordancias pueden ser comunes entre los procesos de socialización en la familia y en el grupo de pares, en los que las formas de representación del rol masculino son más vigiladas y estrictas (Kimmel, 2004). De hecho, los rituales de masculinización propios del período adolescente suelen exigir un énfasis en ciertos rasgos masculinos, como la demostración de valentía o la asunción de riesgos -visible en las elevadas tasas de muertes accidentales en estas cohortes más jóvenes-; un énfasis que se ve atenuado con el paso a la vida adulta, cuando cobran más peso otros rasgos masculinos como la protección y el sustento de sus familias o la independencia y el autocontrol (Rivas, 2005).

Otro de los elementos propios de la socialización secundaria, con una influencia predominante en la configuración de las identidades de género, son los medios de comunicación, Internet y las industrias culturales como el cine, donde en las últimas décadas han florecido representaciones alternativas de masculinidad (McKay, Mikosza y Hutchins, 2005). En lo referente al ámbito virtual, hay que tener en cuenta que la influencia no sólo la ejercen las producciones de los medios digitales, sino también aquellas realizadas por los propios sujetos y compartidas a través de todo tipo de dispositivos (Jiménez Tostón y Aroca Cifuentes, 2019).

Paralelamente a los conflictos producidos por la influencia de los diferentes agentes de socialización secundaria, a lo largo de la vida del individuo pueden surgir tensiones y resistencias, fruto de la relación dialéctica entre el rol y sus propios impulsos biológicos, deseos e intereses. Goffman señala que, en algunos casos, los sujetos resuelven las incompatibilidades entre la exigencia del rol y sus propios intereses a través de 'jugadas' o estrategias en las que "instrumentalizarían los rituales de interacción para alcanzar sus metas" (Galindo, 2015: 24). Mediante una correcta representación de las fórmulas admitidas de cortejo, un muchacho podría, por ejemplo, conseguir una cita o un encuentro sexual con una chica (Galindo, 2015). Además de para alcanzar unas metas específicas, los movimientos discursivos de los sujetos se guían de manera especial -según Goffman (1974)- por la necesidad de acumular 'crédito social' y extraer un valor positivo de nosotros mismos. Es interesante comprobar cómo -al igual que los hombres heterosexuales- los varones homosexuales pueden utilizar en determinados contextos, atributos y comportamientos estereotípicamente masculinos en un intento de acumular 'capital masculino', entendido éste como el poder social y simbólico adquirido al desplegar rasgos y comportamientos asociados a la masculinidad hegemónica (Ravenhill y de Visser, 2017).

\section{METODOLOGÍA}

Para explorar las posibles variaciones en el rol masculino hegemónico tradicional entre los jóvenes varones españoles, llevamos a cabo 4 grupos de discusión mixtos (en los que participaron 13 chicas y 14 chicos) y 4 entrevistas semi-estructuradas con varones residentes en la Comunidad de Madrid. El diseño de la muestra incorporó los perfiles sociodemográficos que podrían funcionar como dimensiones estructurantes de los roles de género: la clase social y la orientación sexual. Dado que la posición social es generadora de sus propios esquemas generativos de prácticas y discursos (Bourdieu, 1988), utilizamos esta dimensión a la hora de formar los grupos. Así, un grupo lo conformó participantes de un centro privado de muy elevado nivel socioeconómico en Villafranca del Castillo; 
dos grupos correspondían a alumnos de institutos públicos y familias de clase media en Arganda del Rey y Madrid; mientras que un cuarto grupo eran estudiantes de un centro público y de origen socioeconómico medio-bajo en Alcorcón. A esta heterogeneidad entre grupos también se han añadido el criterio de heterogeneidad intra-grupo en torno a la dimensión de orientación sexual.

La investigación (exploratoria) se realizó (entre marzo de 2018 y marzo de 2019) a partir de un guión estructurado en torno a las categorías expuestas en la Tabla I y que conforman los rasgos o atributos de la masculinidad hegemónica tradicional: Autosuficiencia e individualidad, Transcendencia y éxito, Papel de proveedor, Heroísmo y violencia, Superioridad sobre las mujeres y Heterosexualidad activa. Para el análisis y codificación de las transcripciones se utilizó el programa de análisis de contenido cualitativo QCAmap.

La técnica de grupos de discusión persiguió captar las opiniones de los sujetos ante las presiones y constricciones externas, pues son en estas situaciones como tienden a producirse en la realidad cotidiana (Martín Criado, 2014). La censura estructural propia de la situación social generada, actuaría favorablemente a la hora de desvelar los rasgos masculinos que gozan de mayor grado de aceptabilidad y legitimidad entre los jóvenes.

El juicio de los iguales es especialmente importante en el caso de las representaciones de género masculinas, consideradas incluso un mero ejercicio de exposición dirigida al resto de los varones (García García, 2010). Como plantea Kimmel, la masculinidad es un constructo que se desarrolla eminentemente en presencia de otros varones, pues son "ellos los que nos miran, nos clasifican, nos conceden aceptación en el reino de la virilidad" (2004: 186). Por su parte, las entrevistas pretendieron captar lo que expresaría un individuo liberado de la presión de esos juicios externos.

Durante el análisis examinamos, también, las contradicciones y los vaivenes en la argumentación de los interlocutores, un aspecto de especial relevancia a la hora de interpretar las dinámicas y estrategias simbólicas inherentes a la representación de cualquier rol social (Martín Criado, 2014). Este análisis reveló en nuestro caso, las ambivalencias a la que se ven sometidos los jóvenes varones, fruto de las tensiones entre el rol socialmente definido y el exigido en diferentes contextos sociales.

La inclusión de chicas en los grupos de discusión permitió, asimismo, indagar en los atributos masculinos predefinidos y valorados por los significantes femeninos. Éstos son de gran importancia, no sólo porque las jóvenes son agentes mediadores de la realidad social, sino porque una interacción exitosa con ellas, se convierte en un activo importante que puede ser mostrado al resto de varones (García García, 2010).

\section{DISCUSIÓN DE RESULTADOS}

\section{El rol masculino dominante entre las nuevas generaciones}

Del análisis de los grupos de discusión y entrevistas se extrae que, con independencia del origen socioeconómico, el rol masculino dominante entre los jóvenes de hoy comparte muchos de los atributos del ideal masculino tradicional con algunas variaciones. Siguen siendo frecuentes entre los varones las manifestaciones de 'Heroísmo y violencia', especialmente en lo que se refiere a las demostraciones de valentía, que a menudo entrañan conductas de riesgo: "Hace un par de semanas me subí en el coche de un amigo y dije ¡Dios, dónde me he metido! Normalmente soy muy de adrenalina de ¡Venga, vamos a hacerlo!" (Varón, 18 años, heterosexual, nivel socioeconómico medio). "Sí que he hecho bastantes veces el cabra, bastante peligrosas (...). Ese momento que estás con tus amigos y te dicen 
¿a que no hay huevos?" (Varón, 17 años, heterosexual, nivel socioeconómico medio). "Sí, a mi me gusta la sensación, ese miedo cuando vas a saltar y luego saltas y ves que sigues vivo" (Varón, 17 años, heterosexual, nivel socioeconómico alto). Los jóvenes a menudo comentan estas hazañas con orgullo y diversión ante sus iguales y consideran algunos comportamientos violentos como preceptos ineludibles: "Quedar por debajo de alguien que es igual a ti y que está retándote, inconscientemente cuesta... no puedes quedar como inferior" (Varón, 17 años, heterosexual, nivel socioeconómico alto). Además, esta característica es de las más repetidas en su definición de hombre: "Ser hombre es ser valiente, fuerte mental y físicamente" (Varón, 18 años, heterosexual, nivel socioeconómico medio). Estas afirmaciones ponen de relieve algunas dinámicas ya descritas por los enfoques psicoanalíticos feministas (Chodorow 2002, citado por García García, 2010) según los cuales, esta propensión masculina a la agresividad es una respuesta defensiva ante una eventual humillación por parte de los iguales.

Igualmente, los adolescentes otorgan gran importancia al valor de la 'Autosuficiencia e independencia', valorando la capacidad de valerse por ellos mismos y prescindiendo de cualquier tipo de apoyo. Al contrario que las chicas, los varones invariablemente manifiestan que siempre intentan resolver sus problemas por sí mismos. "Yo si pido ayuda es cuando ya veo que no tengo otros recursos" (Varón, 17 años, heterosexual, nivel socioeconómico medio). La tendencia a no compartir ni expresar emociones -que escondería en el fondo una incapacidad para demostrar vulnerabilidad-es, además, uno de los atributos más característicos entre los jóvenes: "Yo he llorado una vez en mi vida, dos como mucho, delante de mis amigos" (Varón, 16 años, heterosexual, nivel socioeconómico medio-bajo). "De forma habitual no hablamos de eso [sentimientos], son tus cosas, tus problemas, algo tuyo" (Varón, 17 años, heterosexual, nivel socioeconómico alto).

Este tipo de conductas relativas a no aparecer débiles o vulnerables son en cierta medida reforzadas por sus iguales femeninas, quienes con bastante frecuencia admiten tener en su ideal de pareja un chico 'decido' y 'seguro de sí mismo'. No obstante, las chicas demandan y valoran actitudes contrarias a la masculinidad hegemónica cuando se trata de la esfera íntimo-amorosa. En este terreno las chicas esperan que los chicos se comporten de acuerdo a una serie de prácticas 'románticas' (esquemas estereotipados acerca de las maneras femeninas de abordar los sentimientos amorosos) donde la sensibilidad y la expresión emocional son rasgos deseables, "Me gusta un chico que sea romántico, que me apoye, que sea cariñoso, que sepa escuchar" (Mujer, 17 años, heterosexual, nivel socioeconómico medio). Estos resultados son consistentes con investigaciones previas (Vicente Olmo, 2018) que ya señalaban las relaciones de pareja heterosexuales como el terreno donde, con mayor frecuencia, se solapaban dos mandatos opuestos de lo deseable.

Asimismo, continúa siendo relevante la necesidad de 'Trascendencia, éxito y prestigio social', lo cual puede observarse en los intereses profesionales manifestados por los varones, más vinculados a itinerarios profesionales socialmente más valorados y mejor remunerados, como los ligados al área tecnológica. Además, la mayoría aspira a alcanzar posiciones sociales relevantes y admite una preferencia por el liderazgo: "Me gustaría abrir una empresa y que no me controlen a mí, sino llevarlo a mi modo" (Varón, 18 años, heterosexual, nivel socioeconómico medio), "Me gustaría ser jefe porque me veo con capacidad de liderazgo" (Varón, 16 años, heterosexual, nivel socioeconómico medio). Las predisposiciones observadas son acordes con las tendencias todavía advertidas en torno la elección de itinerarios profesionales entre jóvenes de nuestro país, según las cuales, los varones en niveles de la ESO todavía planean, en mucha mayor medida que las chicas, decantarse por itinerarios STEM, los cuales generalmente proporcionan mejores perspectivas laborales (González Ramos, Vergés Bosch y Martínez García, 2017; López Rupérez y García Expósito, 2018). 
Las características de 'Heterosexualidad activa' que se les presupone a los hombres, siguen por otro lado plenamente vigentes entre las nuevas generaciones. De hecho, aquellos que no encajan con la heteronormatividad propia del rol masculino, admiten haber ocultado su orientación sexual para evitar situaciones de acoso que aseguran haber presenciado con bastante frecuencia: "Así lo más grave, fue antes de salir del armario, que en mi clase había un chico a quien todo el rato le llamaban maricón y se metían con él, y a mí me daba miedo que me hicieran lo mismo. Así que pensé: hay que disimular, o me ligo a una chica, que obviamente no, o me hago el homófobo como ellos. Y empecé a meterme yo también con él. Cada vez que le decían algo, yo también, el que más caña le daba" (Varón, 16 años, gay, nivel socioeconómico bajo). Estas afirmaciones son reflejo de la proliferación de actitudes homófobas en los centros escolares españoles de los que han dado cuenta diferentes estudios (Pichardo, 2009) y, como defiende García García (2010), nos permiten entender de qué manera se desencadenan ciertos comportamientos colectivos, como la participación en casos de acoso escolar, en los que los jóvenes llegan a cometer atrocidades impensables a título individual.

En los grupos de discusión se evidencia, además, que las chicas son mucho más abiertas que los chicos en relación a la diversidad sexual. Mientras que era frecuente encontrar a chicas lesbianas y bisexuales para participar en la investigación -sin que su orientación sexual originara el menor signo de extrañeza o desaprobación entre sus iguales femeninas-, resultó mucho más difícil encontrar chicos de orientaciones sexuales distintas a la heteronormativa para participar en los grupos. Ello parece también indicativo de la preeminencia de la heterosexualidad como uno de los mandatos fundamentales de la masculinidad hegemónica entre los jóvenes. La menor tolerancia de los varones adolescentes en torno a la diversidad sexual en comparación con las chicas, es también consistente con otras investigaciones recientes sobre masculinidades y feminidades llevadas a cabo recientemente en España (Jiménez Tostón y Aroca Cifuentes, 2019) y contrasta con los niveles de tolerancia de la sociedad española en general que, según organismos como la Comisión Europa, estaría entre los más altos de la UE (Rovirosa y Herrera, 2019).

Los jóvenes también responden al precepto de mostrarse activos sexualmente como demostración de virilidad. Además, en general suelen considerar esta tendencia como fruto de una realidad biológica ajena a cualquier aprehensión socialmente entendida, y en modo alguno condicionada: "A mí me parece que los hombres somos más insaciables, más animales, por decirlo así" (Varón, 17 años, heterosexual, nivel socioeconómico alto). "Los hombres nos dejamos llevar mucho más por los instintos" (Varón, 17 años, heterosexual, nivel socioeconómico alto). Sin embargo, en ausencia de vigilancia de sus iguales, reconocen que la tendencia de los chicos a ser más promiscuos puede responder a un intento de ganar crédito social o 'capital masculino': "Es más natural para el chico ser infiel ¿Por qué? Si uno tiene una pareja y se va con otra, queda bien ante sus amigos, cosa que está mal, pero los chicos que son infieles en mi opinión lo hacen para sentirse queridos por sus amigos, para sentirse superiores" (Varón, 17 años, heterosexual, nivel socioeconómico medio).

A pesar de la vigencia de los rasgos masculinos clásicos hasta ahora señalados, también el rol hegemónico tradicional está sufriendo algunas variaciones palpables entre las jóvenes generaciones. El rasgo tradicionalmente masculino que más erosión ha sufrido, es el de 'proveedor'. Parece que el nuevo modelo de hombre es aquel que, además de aportar a la economía familiar, participa activamente en las labores domésticas y de cuidados: "Como padre tendría, por supuesto, las mismas responsabilidades que la madre (...) si les tengo que lavar, ayudar en temas de casa, limpiar, cocinar... pues eso ayudar, bueno ayudar no, hacer en verdad" (Varón, 18 años, heterosexual, nivel socioeconómico medio). Este cambio fundamental viene forzado, sin duda, por dos fenómenos sociales de influencia reciproca: los nuevos valores en torno a la igualdad de género y la incorporación general 
de la mujer al mercado laboral. De hecho, las chicas defienden de manera decidida su vida profesional, que parecen identificar como el mejor garante de la igualdad de género, con lo que le otorgan incluso más importancia que los propios varones. Es interesante observar que, mientras muchos de los sujetos masculinos dicen estar dispuestos a dejar de trabajar una temporada para cuidar de sus hijos o padres, muchas chicas aseguran que no abandonarían sus trabajos para ejercer de cuidadoras: "Buscaría guardería o cualquier alternativa antes de dejar el trabajo" (Mujer, 17 años, bisexual, nivel socioeconómico medio), "Yo no lo haría [dejar de trabajar para cuidar de sus hijos] si no fuera extremadamente necesario" (Mujer, 17 años, heterosexual, nivel socioeconómico alto). "Yo estaría cómodo, si es necesario [con dejar de trabajar para cuidar de sus hijos] (Varón, 17, heterosexual, nivel socioeconómico medio).

Igualmente, el consenso social en torno a la igualdad de género ha erradicado del discurso masculino cualquier alusión acerca de una supuesta 'superioridad frente a las mujeres', lo cual suscitaría un importante rechazo social. El hombre que cuenta con mayor legitimidad social en nuestros días, es aquel que considera a la mujer una igual. Huelga decir que ello no implica que no existan contextos en los que se inste a los varones a mostrar esa superioridad o no se les penalice por aparecer como inferiores. De hecho, en las entrevistas, sí reconocen que 'algunos hombres' (en ningún caso admiten que puedan ser ellos mismos) pueden sentirse incómodos en contextos en los que una mujer ocupe una posición superior: "Si por ejemplo un hombre entra en una empresa y es una mujer la jefa, ya le discriminan a él en plan 'jaja una mujer está por encima de ti', y van a por él. Yo creo que eso les afecta porque, hay tanto machismo, que estar por debajo de una mujer para ellos es como un problema, que es una tontería, pero yo creo que ellos piensan que les perjudica." (Varón, 16 años, heterosexual, nivel socioeconómico medio).

\section{Cuestionamiento, ambivalencias y divergencias ante el rol masculino hegemónico}

Vemos cómo las ambivalencias de los jóvenes son frecuentes. Aunque por un lado la mayoría afirma no identificarse con el arquetipo masculino dominante $-\mathrm{y}$, de hecho, muchos de ellos se definen a sí mismos como feministas- por otro, tienden, como hemos comprobado, a representar muchos de los rasgos del rol patriarcal en sus interacciones diarias. Cuando se les pregunta de manera explícita por este aspecto, defienden una manera flexible de ser hombres acorde con el discurso feminista: "Un hombre puede estar en casa, puede llorar, no tiene por qué ser fuerte y valiente" (Varón, 16 años, heterosexual, nivel socioeconómico mediobajo). Sin embargo, vemos cómo la mayoría de los rasgos de la masculinidad hegemónica tradicional, no sólo se mantienen vigentes, sino que los jóvenes tienden a autorregular su comportamiento para amoldarse a lo que se espera de ellos: "El otro día me vinieron ganas de llorar, pero... pasé (...) se me saltaron un poco las lágrimas, pero me preguntaron qué tal y me dio un poco de corte" (Varón, 17 años, heterosexual, nivel socioeconómico medio). Incluso, aunque en ocasiones defienden una manera de definirse contrarios a la masculinidad hegemónica, ésta muchas veces supone un alarde de los imperativos de independencia y valentía: "Me da igual, yo soy yo mismo, si alguien piensa que no soy un hombre me río de ellos" (Varón, 17 años, heterosexual, nivel socioeconómico medio).

En cuanto a las consecuencias negativas del rol masculino dominante, los jóvenes identifican con bastante frecuencia la dificultad para expresar emociones como el efecto más pernicioso del ideal masculino. En las entrevistas, sin presencia de otros compañeros, los jóvenes admiten con más llaneza sus dificultades en torno a la expresión emocional, un terreno que continúan considerando de dominio femenino: "Lo peor y más complicado de ser un hombre, para mí, es expresarme. Porque solemos ser más cerrados, no expresamos tanto nuestros sentimientos" (Varón, 16 años, heterosexual, nivel socioeconómico bajo). 
"Yo creo que las mujeres sienten más (...) Nos perdemos eso yo creo" (Varón, 17 años, heterosexual, nivel socioeconómico medio).

A pesar de todo, son las chicas las que demuestran ser más conscientes de los perjuicios de una masculinidad tóxica, aludiendo a menudo a la menor intimidad en relaciones de amistad entre hombres, a la dificultad para expresar emociones y a la presión social para mantener su estatus "de machito".

Finalmente, si comparamos las disposiciones en torno al rol de género y las propias del grupo según su posición en la estructura social, no encontramos apenas diferencias entre grupos en función de las variables socioeconómicas tenidas en cuenta. Solamente en lo referente a la variable de orientación sexual existe alguna diferencia. En este sentido, los chicos gais parecen ser algo más conscientes de las consecuencias negativas que la masculinidad hegemónica provoca en los hombres. Ellos identifican la presión por tener un cuerpo normativo o el rechazo hacia aquellos que se dedican a un campo profesional feminizado: A los chicos que (...) no tienen un cuerpo tan perfecto como los que salen en redes sociales, se les suele juzgar mucho" (Varón, 17 años, gay, nivel socioeconómico bajo). "Si un hombre estudia o se dedica a ese mundo [la moda], se le considera más femenino que masculino. Hay chicos que se lo pierden por miedo a la sociedad" (Varón, 17 años, gay, nivel socioeconómico bajo).

Asimismo, todos los sujetos reconocen haber presenciado situaciones de acoso hacia otros varones por el hecho de no reproducir el rol típicamente masculino, lo que evidencia la presión ejercida por los iguales masculinos a la hora de garantizar su cumplimiento: "Con 14, 15 años lo he visto mucho. Cosas como si no eres como nosotros, no entras en el grupo, no juegas con nosotros (...) por cosas como la forma de hablar o los andares" (Varón, 17 años, heterosexual, nivel socioeconómico medio). Estos resultados dan cuenta del fuerte compromiso de los varones por las formas de representación social del rol masculino en entornos homosociales y las graves represalias que se derivan de su no cumplimiento (Kimmel, 2004; García García, 2010).

\section{CONCLUSIONES}

Pese al incipiente interés social en torno a las 'masculinidades tóxicas', no existe un real cuestionamiento entre los jóvenes sobre la representación social dominante de lo masculino, sino más bien prácticas que reproducen el marco preceptivo de la masculinidad hegemónica tradicional, con algunas variaciones también destacables.

Según los datos extraídos de esta investigación, y a pesar de las transformaciones sociales y el discurso culturalmente legitimado en torno a la igualdad de género, muchos de los rasgos del rol hegemónico tradicional masculino siguen predominantemente vigentes entre los adolescentes. El arquetipo masculino que los jóvenes tienen hoy como modelo de referencia en España, y por tanto tienden a reproducir, es aquel varón que reprime emociones que revelan debilidad, se muestra independiente y autosuficiente, se pone en riesgo para mostrar su valor, hace uso legitimado de la violencia, es heterosexual y activo sexualmente. De lo revelado en las entrevistas se vislumbra, de hecho, uno de los aspectos estructurales que contribuyen a la reproducción de las formas dominantes de masculinidad: los esquemas de discurso y acción congruentes con el rol masculino son continuamente vigilados y reforzados por el grupo de iguales. El individuo auto-regula, así, sus propias expresiones y comportamientos debido a la censura estructural, dicho de otro modo, el varón joven encarna el rol prescrito socialmente como modo de evitar la sanción y/o el ostracismo. Esto es observable, en primer lugar, en el hecho de que algunos 
aspectos revelados en las entrevistas -especialmente todo lo referente a los malestares experimentados, o cuestionamientos que hacen del rol-, quedaban más ocultos en los grupos de discusión debido a la vigilancia externa. Además, todos los chicos invariablemente admitían haber presenciado situaciones de acoso hacia quienes se distanciaban de los esperable en un varón, y algunos admitían, incluso, haber reprimido o forzado expresiones, actitudes 0 acciones para adecuarse a la norma.

La investigación sí arroja variaciones en uno de los atributos tradicionalmente masculinos como es el rol de proveedor, que hoy también asumen las mujeres. De hecho, las jóvenes de hoy no parecen dispuestas a ceder en este nuevo papel que consideran una conquista social del feminismo y quizá por ello, sean menos favorables a sacrificarlo para ejercer de cuidadoras que los chicos. Éstos, por su parte, deben responder a las demandas de sus referentes femeninas en cuestiones como el reparto equitativo de las tareas domésticas y la labor de crianza. También ha perdido legitimidad cualquier discurso que pueda sugerir una superioridad masculina. No obstante, aunque los chicos niegan invariablemente que los hombres deban ocupar ese lugar preponderante frente a las mujeres, en las entrevistas sí admiten la existencia de cierta presión social por no aparecer como inferiores a ellas.

El nuevo rol masculino tampoco es ajeno a contradicciones y ambivalencias. Mientras que si se les pregunta directamente, la mayoría de los jóvenes defienden un modelo de hombría que desafía los preceptos más tradicionales, en muchas de sus respuestas y actitudes encontramos en realidad un intento más o menos consciente de reproducir la masculinidad hegemónica clásica. Parte de esta ambivalencia está producida por la discrepancia entre los nuevos valores de igualdad y la necesidad de responder a diferentes expectativas de comportamiento culturalmente compartidas por el grupo de iguales en diferentes contextos situacionales. En lo relativo a la influencia ejercida por las referentes femeninas, es interesante observar por ejemplo cómo, al mismo tiempo que las chicas demandan varones sensibles sobre todo en el ámbito amoroso, siguen prefiriendo -y de este modo condicionando- chicos no dependientes y seguros de sí mismos.

En cuanto a los efectos negativos del rol que se les impone socialmente, los jóvenes admiten experimentar malestares y se sienten así mismos impedidos sobre todo en los aspectos relacionados con la represión emocional. La necesidad de no mostrar sentimientos que puedan revelar debilidad es una de las imposiciones del ideal masculino que perciben como más alienantes y quizá el coste asociado a cumplir con dicho ideal, del que son más conscientes.

\section{REFERENCIAS BIBLIOGRÁFICAS}

Austin J. (1971). Cómo hacer cosas con palabras. Palabras y acciones. Barcelona: Paidós.

Azpiazu Carballo, J. (2017). Masculinidades y feminismo. Barcelona: Virus Editorial.

Ballesteros, J. C., Rubio, A., Sanmartín, A. y Tudela, P. (2019). Barómetro Juventud y Género 2019. Identidades y representaciones en una realidad compleja. Centro Reina Sofía sobre Adolescencia y Juventud, Fad. (en línea)

http://www.adolescenciayjuventud.org/que-hacemos/monografias-y-estudios/ ampliar.php/Id_contenido/127043/. Acceso 28 de febrero de 2020.

Bauman, Z. (2005). Amor líquido. Acerca de la fragilidad de los vínculos humanos. Buenos Aires: Fondo de Cultura económica.

Ballester Brage, Ll. y Orte Socias, C. (2019). Nueva pornografía y cambios en las relaciones interpersonales (En línea) https://cdn.20m.es/adj/2019/06/10/4007.pdf. Acceso 11 de octubre de 2019. 
Beck, U. y Beck-Gernsheim, E. (2001). El normal Caos del amor. Barcelona: Paidós Ibérica.

Berger, P. y Luckmann, T. (1968). La construcción social de la realidad. Buenos Aires: Amorrortu.

Bridges, T. y Pascoe, C. J. (2014). Hybrid masculinities: New directions in the sociology of men and masculinities. Sociology Compass, 8(3), 246-258. https://doi.org/10.1111/soc4.12134

Butler, J. (2001). El género en disputa. México: Paidós.

Bonino, L. (2002). Masculinidad, salud y sistema sanitario: el caso de la violencia masculina, Seminario de la OMS/Oficina europea: Mainstreaming de género en las políticas de salud en Europa. (En línea).

http://img1.oaistore.com/ipes/img/00014001_00014500/00014061/descarga/ 00014061_0001.pdf. Acceso, 18 de abril de 2019

Bonino, L. (2003). Masculinidad hegemónica e identidad masculina, Dossiers Feministes, 6. Editado por el Seminario de Investigación Feminista de la Universitat Jaume I de Castellón, España. (En línea).

https://www.raco.cat/index.php/DossiersFeministes/article/viewFile/102434/153629. Acceso, 18 de abril de 2019

Bourdieu, P. (1988). La distinción. Criterios y bases sociales del gusto. Madrid: Taurus.

Bourdieu, P. (2000). La dominación masculina. Barcelona: Anagrama.

Castells, M. y Subirats, M. (2007). Mujeres y hombres: ¿Un amor imposible? Madrid: Alianza.

Chodorow, N. (2002). The enemy outside: thoughts on the psycho-dynamics of extreme violence with special attention to men and masculinity. En Gardiner, Judith K. (Ed.), Masculinity Studies and Feminist Theory. New Directions (pp. 235-260). Columbia University Press, New York.

Connell, R.W. (2000). The Men and the Boys. Berkeley: University of California Press.

Connell, R. W. y Messerschmidt, J. (2005). Hegemonic Masculinity: Rethinking the Concept. Gender \& Society, 19(6), 829-859. https://doi.org/10.1177/0891243205278639

Derrida, J. (1988). Limited Inc. Evanston, IL: Northwestern University Press.

Duque, C. (2010). Judith Butler y la teoría de la performatividad de género. Revista Educación \& pensamiento, 85-95.

Fordice, D. J. y Nielsen, L. E. (2013). Constructing Gender: Contradictions in the Life of a Male Elementary Teacher. (En línea). https://files.eric.ed.gov/fulltext/EJ1098410.pdf. Acceso, 26 de abril de 2019

Foucault, M. (2003). Historia de la Sexualidad 2. El uso de los placeres. Buenos Aires: Siglo XXI Editores Argentina SA.

Galindo, J. (2015). Erving Goffman y el orden de la interacción. Acta sociológica, 66, 11-34. https://doi.org/10.1016/j.acso.2014.11.002

García García, A. A. (2010). Exponiendo hombría. Los circuitos de la hipermasculinidad en la configuración de prácticas sexistas entre varones jóvenes. Revista de Estudios de Juventud, 10(89), 59-78. (En línea) http://www.injuve.es/sites/default/files/revista89_3.pdf. Acceso 28 de febrero de 2020.

Goffman, E. (1981). La Presentación de la persona en la vida cotidiana. Buenos Aires: Amorrurtu. 
Goffman, E. (1974). Frame Analysis. An Essay on the Organization of Experience, Nueva York: Harper \& Row (reed. Boston, Northeastern University Press, 1986).

González, M. G. (2006). Estrés laboral, afrontamiento y sus consecuencias: el papel del género (Tesis doctoral). Universitat de Valencia, Valencia. https://www.uv.es/maglogon/GonzalezMorales2006TESIS.pdf

González Ramos, A. M., Vergés Bosch, N. y Martínez García, J. S. (2017). Las mujeres en el mercado de trabajo de las tecnologías. REIS, 159, 73-90. https://doi.org/10.5477/cis/reis.159.73

Instituto Nacional de Estadística-INE. (2018). Estadística de Condenados (en línea) https://www.ine.es/ss/Satellite?_Les_

Instituto Nacional de Estadística-INE. (2019). Defunciones según la causa de muerte 2017. Suicidios por edad y sexo. (En línea).

https://www.ine.es/jaxi/Tabla.htm?path=/t15/p417/a2017//0/\&file=05001.px\&L=0. Acceso, 11 de octubre de 2019.

Instituto Nacional de Estadística-INE. (2019). Indicadores de mortalidad 2018. Esperanza de vida al nacimiento según sexo. (En línea)

https://www.ine.es/jaxiT3/Tabla.htm?t=1414. Acceso, 11 de octubre de 2019.

Instituto Nacional de Estadística-INE. (2019). Estadística de Nulidades, Separaciones y Divorcios (ENSD) Año 2018. (En línea). https://www.ine.es/prensa/ensd_2018.pdf. Acceso, 11 de octubre de 2019.

Instituto Nacional de Estadística-INE. (2019). Defunciones según la causa de muerte 2017. Distribución de la mortalidad por causas, sexo y edad. (En línea).

https://www.ine.es/jaxi/Tabla.htm?path=/t15/p417/a2017//0/\&file=01003.px\&L=0. Acceso, 11 de octubre de 2019.

Instituto Nacional de Estadística-INE. (2019). Condenados por delitos sexuales. Delitos sexuales según sexo. (En línea). https://www.ine.es/jaxiт3/Tabla.htm?t=28750\&L=0. Acceso, 11 de octubre de 2019.

Jiménez Tostón, G. y Aroca Cifuentes, F. J. (2019). Masculinidades y feminidades en preadolescentes residentes en contextos rurales de Castilla-La Mancha. Revista de Sociología de la Educación (RASE), 12(1), 40-62. https://doi.org/10.7203/RASE.12.1.13253

Jurado, T. y Bueno, X. (2019). Avanzando hacia los permisos parentales igualitarios en España. Una conversación con Teresa Jurado-Guerrero, Encrucijadas. Revista Crítica de Ciencias Sociales, 18, e1801.

Kimmel, M. S. (2004). "Masculinity as Homophobia: Fear, Shame, and Silence in the Construction of Gender Identity." En P. F. Murphy (Ed.) Feminism and Masculinities (pp.182-99). Oxford: Oxford University Press.

López Rupérez, F. y García García, I. (2018). PISA 2015 y las CCAA. Diagnósticos empíricos y políticas de mejora. Universidad Camilo José Cela. (En línea)

https://www.ucjc.edu/wp-content/uploads/pisa2015_ccaa.pdf. Acceso, 28 de febrero de 2020.

Mac an Ghaill, M. y Haywood, C. (2012). Understanding boys': thinking through boys, masculinity and suicide. Social Science \& Medicine, 74, 482-489.

https://doi.org/10.1016/j.socscimed.2010.07.036 
McKay, J., Mikosza, J. y Hutchins, B. (2005). Gentlemen, the lunchbox has landed. Representations of masculinities and men's bodies in the popular media. En R. W. Connell, M. Kimmel (Eds.). Handbook of Studies on Men and Masculinities (pp. 270-288). Thousand Oaks, CA: Sage. https://doi.org/10.4135/9781452233833.n16

Martín Criado, E. (2014). Mentiras, inconsistencias y ambivalencias. Teoría de la acción y análisis del discurso. Revista Internacional de Sociología, 72(1), 115-138. https://doi.org/10.3989/ris.2012.07.24

Mead, G. H. (1973). Espíritu, persona y sociedad. Barcelona: Paidós Básica.

Meil Landwerlin, G. (2017). Permisos parentales para hombres y corresponsabilidad en el cuidado de niños. Revista del Ministerio de Empleo y Seguridad Social, (131), 15-34.

Merton, R. K. (1980). Ambivalencia sociológica y otros ensayos. Madrid: Espasa Calpe.

Ministerio de Presidencia, Relaciones con las Cortes e Igualdad. (2019). Ficha estadística de víctimas mortales por violencia de género. (En línea).

http://www.violenciagenero.igualdad.mpr.gob.es/violenciaEnCifras/victimasMortales/ fichaMujeres/pdf/31_12_2018.pdf. Acceso, 11 de octubre de 2019.

Ministerio de Sanidad, Servicios Sociales e Igualdad. (2019). XXI Encuesta sobre alcohol y otras drogas en España 2017-2018. (En línea).

https://cppm.es/10-12-2018-xii-encuesta-sobre-alcohol-y-otras-drogas-en-espanaedades-2017-2018/. Acceso, 11 de octubre de 2019.

Moen, P. y Orrange, R. M. (2002). Careers and lives: Socialization, structural lag, and gendered ambivalence. Advances in Life Course Research, 7, 231-260. https://doi.org/10.1016/S1040-2608(02)80036-3

Muñoz Sánchez, H. (2017). Hacerse hombres. La construcción de masculinidades desde las subjetividades. Medellín: Universidad de Antioquia. https://doi.org/10.2307/j.ctt21kk1jd

Navarro, E. (2004). Género y relaciones personales íntimas. En E. Barberá, I. Martínez (Eds.), Psicología y género. Madrid: Pearson Educación S.A.

Pichardo Galán, J. I. (2009). Adolescentes ante la diversidad sexual. Catarata: Madrid.

Pichardo Galán, J. I. et. al (2015). Diversidad sexual y convivencia: Una oportunidad educativa. Madrid: Universidad Complutense de Madrid. (En línea). http://www.felgtb.org/rs/3660/d112d6ad-54ec-438b-9358-4483f9e98868/234/ filename/informe-final-diversidad-y-convivencia.pdf. Acceso, 27 de abril de 2019.

Ramírez, S. (2011). El género como actuación. Temas Sociales, 31, 245-259. (En línea). https://bit.ly/2VTKTdb. Acceso, 19 de abril de 2019.

Ravenhill, J. P. y de Visser, R. O. (2017). There are too many gay categories now: Discursive constructions of gay masculinity. Psychology of Men \& Masculinity, 18(4), 321-330. https://doi.org/10.1037/men0000057

Rivas, E. (2005). ¿El varón como factor de riesgo? Masculinidad y mortalidad por accidentes y otras causas violentas en la sierra de Sonora. Estudios Sociales, 13(26), 28-66. (En línea). http://www.redalyc.org/pdf/417/41702602.pdf. Acceso, 31 de marzo de 2020.

Rovirosa, D. y Herrera, E. (2019). El 81\% de los españoles apoyaría un presidente del Gobierno LGTMI. La Vanguardia. (En línea).

https:// www.lavanguardia.com/vida/20190927/47649864887/eurobarometrodiscriminacion-lgbti-encuesta-ue.html. Acceso, 1 de marzo de 2020. 
Sanz, C. (2016). Identidades masculinas: estereotipos de género y participación en la educación física, la actividad física y el deporte (Tesis doctoral). Madrid: Universidad Complutense de Madrid. (En línea). https://eprints.ucm.es/40440/. Acceso, 19 de abril de 2019.

Sargent, P. (2000). Real men or real teachers? Contradictions in the lives of men elementary teachers. Men and Masculinities, 2(4), 410-433.

https://doi.org/10.1177/1097184X00002004003

Segato, R. L. (2018). Contra-pedagogías de la crueldad. Buenos Aires: Prometeo Libros.

Valdés, I. (28 de mayo de 2019). La violencia machista crece más entre los hombres jóvenes. El País.com. (En línea). https://elpais.com/sociedad/2019/05/28/actualidad/1559032717_019231.html. Acceso, 28 de mayo de 2019.

Venegas, M. (2018). El romance adolescente. Un análisis sociológico de la política afectivosexual en la adolescencia. Papers, 103(2), 255-279.

https://doi.org/10.5565/rev/papers.2213

Vicente Olmo, A. (2018). Detalles, frustraciones y desconciertos: El trabajo emocional en las parejas jóvenes heterosexuales. Revista Española de Sociología (RES), 27(1), 67-81. https://doi.org/10.22325/fes/res.2018.4

\section{NOTA BIOGRÁFICA}

Mayra Martínez Avidad es profesora contratada doctora (acreditada por la ANECA) en la Facultad de Educación de la Universidad Camilo José Cela. Doctora en Ciencias de la Comunicación y Sociología por la Universidad Complutense de Madrid. Estancia de investigación de dos años en la Universidad de California, San Diego (UCSD), donde completó diferentes seminarios de formación y desarrolló actividades de investigación y docencia. Colaboradora honorífica del departamento de Sociología VI, Opinión Pública y Cultura de Masas de la UCM.

Alba Pérez López es graduada en Pedagogía por la Universidad Complutense de Madrid. Máster en Formación para el Profesorado en la especialidad de Orientación Educativa por la Universidad Camilo José Cela. Trabajo Fin de Máster consistente en la investigación "Masculinidad: vigencia y evolución del rol de género", con el que obtuvo la calificación de sobresaliente. 
